01;03

\title{
Энергетическая оценка смачивания проводящей каплей заряженной диэлектрической поверхности
}

\author{
(C) А.В. Голочалова, Э.Б. Кулумбаев \\ Белгородский государственный национальный исследовательский университет, Белгород, Россия \\ ฯ E-mail: kulumbaev@bsu.edu.ru
}

Поступило в Редакцию 27 апреля 2021 г.

В окончательной редакции 16 мая 2021 г.

Принято к публикации 16 мая 2021г.

\begin{abstract}
Выполнена энергетическая оценка смачивания проводящей каплей диэлектрической подложки, заряженной фиксированным на ней сторонним электрическим зарядом. Получено, что смачиваемость такой поверхности уменьшается, если средняя плотность индуцированного в основании капли заряда превышает пороговое значение.
\end{abstract}

Ключевые слова: смачивание, проводящая жидкость, электрический заряд, краевой угол.

DOI: 10.21883/PJTF.2021.17.51379.18845

Смачивание как объект исследования представляет научный и практический интерес. В частности, актуальной остается задача управления смачиванием для различных технических приложений. В схеме электросмачивания на диэлектрике для увеличения его смачиваемости жидкостью применяется электрическое поле электродов [1]. Обратный эффект - уменьшение смачивания - наблюдается при размещении проводящей капли на предварительно заряженной диэлектрической подложке [2]. В настоящей работе закономерности смачивания в условиях эксперимента [2] изучаются на основе энергетического подхода.

Рассматривается равновесное осесимметричное состояние капли проводящей жидкости в газе, лежащей на горизонтальной твердой диэлектрической подложке, равномерно заряженной фиксированным на ее ограничивающей плоскости сторонним электрическим зарядом с известной поверхностной плотностью $\sigma_{0}$ (рис. 1 ). В электрическом поле этого заряда происходит поляризация физически однородных фаз. Поэтому индуцированные и связанные заряды появятся только на разделяющих их границах, которые рассматриваются в приближении бесконечно тонких поверхностей. Распределение суммарного (стороннего, индуцированного и связанного) заряда на этих поверхностях на основе подхода Гринберга [3] определяется системой интегральных уравнений

$$
\begin{gathered}
\sigma_{S L}=2 \varepsilon_{0} E_{S L}\left(\sigma_{L G}\right), \\
\sigma_{S G}=\frac{2}{\varepsilon_{G}+\varepsilon_{S}} \sigma_{0}-2 \varepsilon_{0} \frac{\varepsilon_{S}-\varepsilon_{G}}{\varepsilon_{S}+\varepsilon_{G}} E_{S G}\left(\sigma_{L G}\right), \\
\sigma_{L G}=2 \varepsilon_{0}\left[E_{L G}\left(\sigma_{L G}\right)+E_{L G}\left(\sigma_{S L}\right)+E_{L G}\left(\sigma_{S G}\right)\right],
\end{gathered}
$$

где $\sigma$ - поверхностная плотность заряда; $\varepsilon-$ диэлектрическая проницаемость; $E$ - проекция на нормаль (внешнюю на поверхности капли и противоположную орту оси $z$ на границе $S G$ ) к элементу контактной поверхности вектора напряженности электрического поля, создаваемого всеми расположенными вне этого элемента зарядами; $\varepsilon_{0}$ - электрическая постоянная. Здесь и далее нижний одинарный индекс обозначает принадлежность величины к фазе, двойной — к межфазной границе (рис. 1).

Стационарное устойчивое состояние рассматриваемой системы реализуется в минимуме ее энергии $W$. Капля полагается малой настолько, что влиянием силы тяжести на ее форму пренебрегается по сравнению с действием поверхностного натяжения. Тогда $W$ складывается из поверхностной $W_{s}$ и электрической $W_{e}$ энергий:

$$
\begin{aligned}
& W_{s}=\alpha_{L G} A_{L G}+\alpha_{S L} A_{S L}+\alpha_{S G} A_{S G}, \\
& W_{e}=0.5 \varphi_{L} q_{L}+0.5 \int \varphi_{S G} \sigma_{S G} d A .
\end{aligned}
$$

Здесь $\alpha-$ коэффициент поверхностного натяжения на контактной границе фаз с площадью $A ; \varphi-$ скалярный потенциал в месте нахождения заряда $\sigma d A$.

Суммарный заряд $q_{L}=\int \sigma_{L G} d A+\int \sigma_{S L} d A$ на поверхности капли с использованием формул $\sigma_{L G}=\sigma_{L G}^{i} / \varepsilon_{G}$, $\sigma_{S L}=\left(\sigma_{0}+\sigma_{S L}^{i}\right) / \varepsilon_{S}$ (где $\sigma^{i}-$ плотность индуцированного заряда проводника на границе с диэлектриком, причем $\left.\int \sigma_{L G}^{i} d A+\int \sigma_{S L}^{i} d A=0\right)$ приводится к виду

$$
q_{L}=\left[1 / \varepsilon_{S}+\left(1 / \varepsilon_{G}-1 / \varepsilon_{S}\right) \gamma\right] \sigma_{0} A_{S L}
$$

Здесь $\gamma=-\int \sigma_{S L}^{i} d A / \sigma_{0} A_{S L}$ - отношение средней плотности наведенного в основании $S L$ заряда к плотности $\sigma_{0}$ с учетом противоположности знаков этих зарядов.

Задача нахождения формы капли, обеспечивающей минимум $W$ с учетом (1)-(3) при заданном объеме $V$ жидкости, может быть решена только в результате тонкого численного расчета из-за особенностей плотности заряда и электрического поля на линии контакта трех фаз. Поэтому для оценки смачивания задача упрощается. Мениск малой капли полагается сферическим. При $V=2 \pi R_{0}^{3} / 3\left(R_{0}\right.$ - радиус полушара с объемом $\left.V\right)$ 


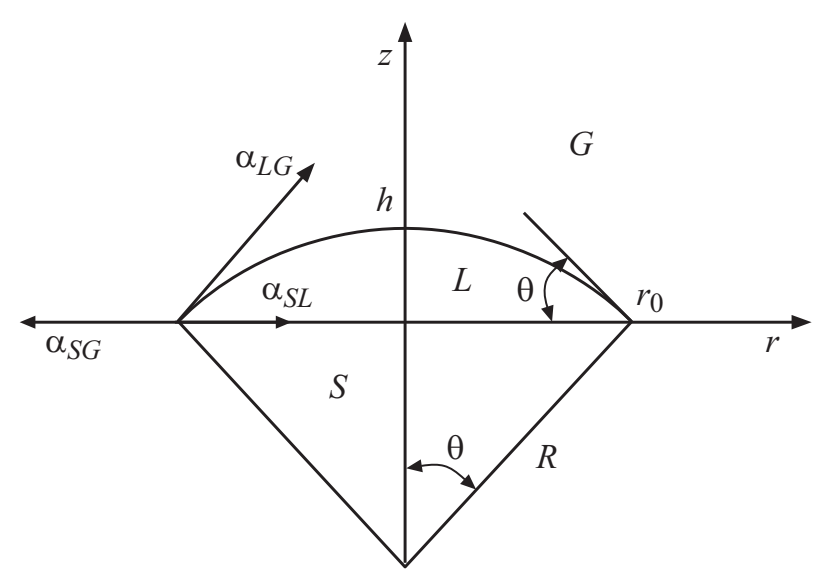

Рис. 1. Геометрия трехфазной системы. $S-$ твердое тело, $L-$ жидкость, $G$ - газ.

шаровой сегмент задается краевым углом $\theta$ (рис. 1) в известных геометрических формулах для радиусов кривизны $R=R_{0} f(\theta)$, основания $r_{0}=R \sin \theta$, высоты $h=R(1-\cos \theta)$ и площадей $A_{L G}=2 \pi R h, A_{S L}=\pi r_{0}^{2}$, где $f(\theta)=\left[2 /\left(2-3 \cos \theta+\cos ^{3} \theta\right)\right]^{1 / 3}$. На незаряженной подложке $\left(\sigma_{0}=0\right)$ минимизация $W_{s}$ дает формулу Юнга [4]: $\alpha_{S G}=\alpha_{S L}+\alpha_{L G} \cos \theta_{Y}$. Тогда обезразмеренная с масштабом $2 \pi R_{0}^{2} \alpha_{L G}$ переменная часть энергии $W_{s}$ из первого выражения (2) записывается следующим образом:

$$
W_{s}^{\prime}=\left(1-\cos \theta-0.5 \sin ^{2} \theta \cos \theta_{Y}\right) f^{2}(\theta) .
$$

Потенциал $\varphi_{L}$ проводника находится методом среднего потенциала [5], результаты которого с приемлемой для оценки интегральных величин точностью согласуются с экспериментальными данными. Сначала рассчитывается потенциал в каждой точке поверхности капли от зарядов на всех контактных границах. Для этого полагается, что распределение индуцированного заряда равномерно и на границе $L G$, и на границе $S L$. Поскольку в основании капли средняя плотность индуцированного заряда $-\gamma \sigma_{0}$, имеем $\sigma_{S L}=(1-\gamma) \sigma_{0} / \varepsilon_{S}$. Для суммарного заряда на мениске получается $\sigma_{L G}=\left(\gamma \sigma_{0} / \varepsilon_{G}\right) \cos ^{2}(\theta / 2)$. Плотность $\sigma_{S G}$ вычисляется по соответствующей формуле (1). Затем вычисленный потенциал усредняется по поверхности капли, и этот средний потенциал поверхности принимается за $\varphi_{L}$. Аналитического результата такой расчет не допускает. Поэтому $\varphi_{L}$ находится численно. Образующая формы капли составляется из дуги окружности радиуса $R$, вырезаемой центральным углом $\theta$, и радиуса $r_{0}$ основания капли (рис. 1). Дуга разбивается на $N$ равных частей. При вращении дуги вокруг оси симметрии образуются заряженные коаксиальные круговые пояски, потенциал каждого из которых отождествляется с известным полем кольцевого заряда $\Delta q_{L G}=\sigma_{L G} 2 \pi r_{n} R \theta / N$, проходящего через центр пояска с координатами $\left(r_{n}, z_{n}\right)$ : $r_{n}=R \sin \theta_{n}, \quad z_{n}=R\left(\cos \theta_{n}-\cos \theta\right), \theta_{n}=\theta(n-0.5) / N$,
$1 \leqslant n \leqslant N$. Поверхностный заряд в основании капли аппроксимируется системой кольцевых зарядов $\Delta q_{S L}=\sigma_{S L} 2 \pi r_{n} R[\sin (\theta n / N)-\sin (\theta(n-1) / N)]$. Аналогично на границе $S G$ : $\Delta q_{S G}=\sigma_{S G} 2 \pi r_{m+0.5}\left(r_{m+1}-r_{m}\right)$, $r_{m}=r_{0}+\left(5 R_{0}-r_{0}\right) m / M, 0 \leqslant m \leqslant M$. Поэтому в произвольной точке $(r, z)$ потенциал имеет вид

$$
\begin{aligned}
2 \pi^{2} \varepsilon_{0} \varphi(r, z)=\sum_{n=1}^{N} & {\left[\frac{\Delta q_{L G}}{R_{1 n}} \mathrm{~K}\left(\frac{2 \sqrt{r r_{n}}}{R_{1 n}}\right)+\frac{\Delta q_{S L}}{R_{2 n}} \mathrm{~K}\left(\frac{2 \sqrt{r r_{n}}}{R_{2 n}}\right)\right] } \\
& +\sum_{m=1}^{M} \frac{\Delta q_{S G}}{R_{2 m}} \mathrm{~K}\left(\frac{2 \sqrt{r r_{m}}}{R_{2 m}}\right)
\end{aligned}
$$

где

$$
R_{1 n}=\sqrt{\left(r+r_{n}\right)^{2}+\left(z-z_{n}\right)^{2}}, R_{2 n}=\sqrt{\left(r+r_{n}\right)^{2}+z^{2}}
$$

$\mathrm{K}$ - полный эллиптический интеграл первого рода.

Для усреднения по поверхности капли, обходя особенность $\mathrm{K}(1)=\infty$, по формуле (5) находим $\varphi$ в точках с координатами $r_{l}=R \sin \theta_{l}$; $z_{l}=R\left(\cos \theta_{l}-\cos \theta\right)$ (для точек основания $\left.z_{l}=0\right)$, где $\theta_{l}=\theta l / N, \quad 0 \leqslant l \leqslant N$, а интегралы для среднего потенциала $\varphi_{L}=\left(\int \varphi_{L G} d A+\int \varphi_{S L} d A\right) /\left(A_{L G}+A_{S L}\right)$ капли и второго слагаемого в энергии $W_{e}(2)$ вычисляются по формуле трапеций.

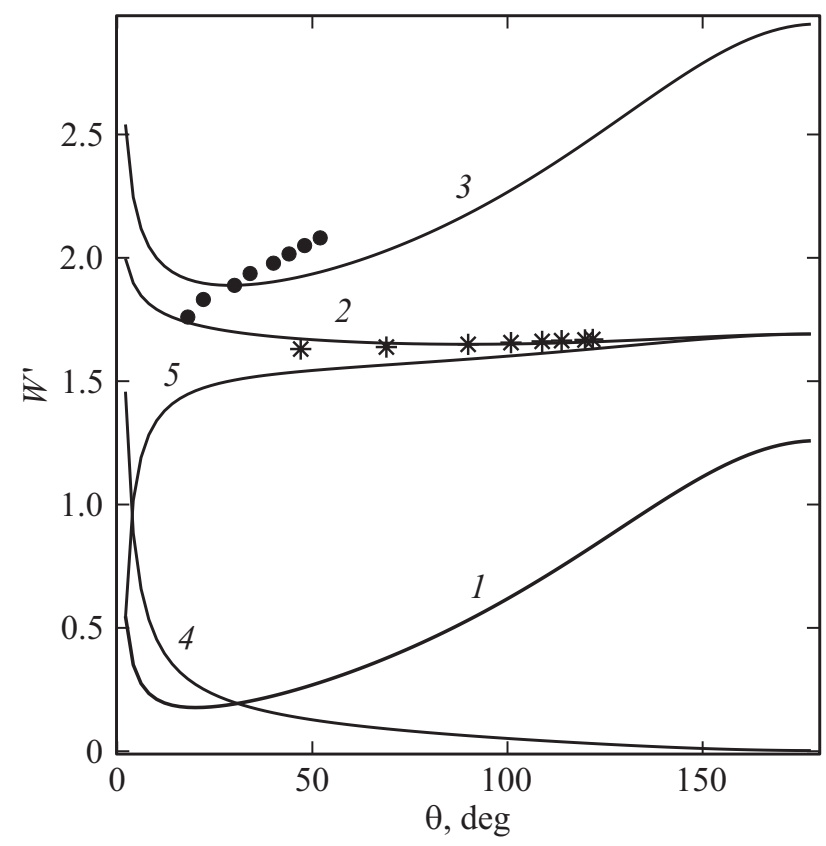

Рис. 2. Зависимости энергии $W_{s}^{\prime}(1)$, полной энергии $W_{e}^{\prime}(2)$ и ее слагаемых в формуле $(6)(4,5)$, а также $W_{s}^{\prime}+W_{e}^{\prime}(3)$ от краевого угла при $\beta=0.1, \gamma=0.5$. Звездочками отмечены минимумы $W_{e}^{\prime}$ при $\gamma=0.36,0.4$ и далее с шагом 0.1 до 1 , кружками обозначены минимумы $W_{s}^{\prime}+W_{e}^{\prime}$ при $\gamma$ от 0.3 до 1 с шагом 0.1 . 
Электрическая энергия $W_{e}$, обезразмеренная с тем же масштабом, что и (4), записывается в виде

$$
W_{e}^{\prime}=0.5 \beta\left(\varphi_{L}^{\prime} q_{L}^{\prime}+\int \varphi_{S G}^{\prime} \sigma_{S G}^{\prime} d A^{\prime}\right),
$$

где $\beta=\sigma_{0}^{2} R_{0} / 4 \varepsilon_{0} \alpha_{L G}-$ отношение характерных значений электрической (с масштабами $\sigma_{0} R_{0} / 2 \varepsilon_{0}$ для потенциала и $\sigma_{0} \pi R_{0}^{2}$ для заряда) и поверхностной энергий.

Расчет проводится при фиксированных значениях $\varepsilon_{G}=1, \varepsilon_{S}=4, \theta_{Y}=20^{\circ}$, близких к экспериментальным данным [2] для капли воды на аморфной пленке диоксида кремния, а параметры $\gamma$ и $\beta$ варьируются. Отметим, что выражение параметра $\gamma$ модели может быть найдено для полусферической капли. Поскольку электрическое поле в основании равномерно заряженной полусферы однородно и равно $\sigma_{L G} / 4 \varepsilon_{0}$, из (1) следует $\sigma_{S L}=\sigma_{L G} / 2$, что дает $\gamma=1 /\left(1+0.25 \varepsilon_{S} / \varepsilon_{G}\right)$.

При фиксированном значении $\beta=0.1$ существует пороговое значение $\gamma \approx 0.35$, ниже которого точка минимума энергии практически совпадает с заданным углом Юнга (рис. 2). При превышении порогового значения краевой угол $\theta$ увеличивается (кружки на рис. 2) и при $\gamma \rightarrow 1$ достигает величины $52^{\circ}$. Пороговое значение $\gamma$ разделяет монотонные и немонотонные зависимости $W_{e}^{\prime}(\theta)$, минимумы которых (звездочки на рис. 2) зависят только от $\gamma$. Сами минимумы обусловлены противоположным характером изменения слагаемых $W_{e}^{\prime}$ в (6) (кривые 4 и 5 на рис. 2). При фиксированном $\gamma$ с ростом $\beta$ по мере уменьшения вклада $W_{s}^{\prime}$ в профиль энергии системы краевой угол $\theta$ монотонно увеличивается и при условии $W_{e}^{\prime} \gg W_{s}^{\prime}$ достигает насыщения в точках минимума $W_{e}^{\prime}$, не зависящих от $\theta_{Y}$. На рис. 2 этому отвечает смещение любого из „кружков“ в соответствующую ему „звездочку““ при $\beta \rightarrow \infty$.

Физическое объяснение рассчитанного увеличения краевого угла следует из эквивалентности влияния электрического заряда на межфазной границе уменьшению поверхностного натяжения. В схеме электросмачивания заряд преимущественно находится на границе $S L$, что приводит к уменьшению $\alpha_{S L}$ и увеличению смачивания. В рассматриваемом случае заряд есть на всех межфазных границах. Вблизи контактной линии трех фаз, где $E_{S G}\left(\sigma_{L G}\right) \approx E_{S L}\left(\sigma_{L G}\right)$, из (1) следует, что

$$
\sigma_{S G} / \sigma_{S L}=\left[(1+\gamma) /(1-\gamma)+\varepsilon_{G} / \varepsilon_{S}\right] /\left(1+\varepsilon_{G} / \varepsilon_{S}\right)>1 .
$$

Поэтому $\alpha_{S G}$ уменьшается больше, чем $\alpha_{S L}$. В результате баланс натяжений будет выполняться на меньшем периметре смачивания с бо́льшим краевым углом независимо от изменения $\alpha_{L G}$ при $\theta \leqslant 90^{\circ}$. Такая закономерность смачивания проводящей каплей заряженной диэлектрической подложки качественно согласуется с экспериментально наблюдаемой в [2].

\section{Конфликт интересов}

Авторы заявляют, что у них нет конфликта интересов.

\section{Список литературы}

[1] F. Mugele, J. Heikenfeld, Electrowetting: fundamental principles and practical applications (Wiley-VCH, 2018), p. 133-136.

[2] D. Aronov, M. Molotskii, G. Rosenman, Appl. Phys. Lett., 90 (10), 104104 (2007). DOI: 10.1063/1.2711656

[3] Г.А. Гринберг, Избранные вопросы математической теории электрических и магнитных явлений (Изд-во АН СССР, М.-Л., 1948), с. 255-260.

[4] Б.Д. Сумм, Ю.В. Горюнов, Физико-химические основы смачивания и растекания (Химия, М., 1976), с. 15-16.

[5] Ю.Я. Иоссель, Э.С. Кочанов, М.Г. Струнский, Расчет электрической емкости (Энергоиздат, Л., 1981), c. $24-31$. 